\title{
Pertinencia de los modelos multivariantes en medicina de urgencias
}

\author{
Rodolfo Rivas-Ruiz" ${ }^{1,2 *}$,Karla M. Silva-Jivaja², Jacquelin L. Galicia-Solórzano², Kevin F. Ríos-Brito², \\ Paola Zamora ${ }^{3}$, Marcela Pérez-Rodríguez ${ }^{1}$ e Ivonne A. Roy-García ${ }^{1}$ \\ ${ }^{1}$ Coordinación de Investigación en Salud, Centro de Adiestramiento e Investigación Clínica, Instituto Mexicano del Seguro Social, Población, Ciudad

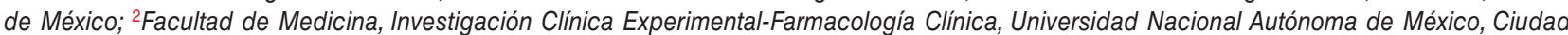 \\ de México; ${ }^{3}$ Departamento de Ortopedia, Centro Médico ABC, Campus Santa Fe, Ciudad de México. México
}

\begin{abstract}
Resumen
En medicina, los modelos de análisis multivariante se emplean para estimar el efecto de variables de interés, tomando en consideración su relación con múltiples factores (confusores o modificadores del efecto) que pudieran predecir en conjunto un desenlace o resultado. Los modelos multivariantes que se emplean con mayor frecuencia en la investigación clínica son: la regresión lineal $(R L)$, la regresión logística (Rlog) y el modelo de riesgos proporcionales de Cox (MRPC). Estos modelos se deben de establecer previamente en el protocolo a partir del desenlace o resultado esperado en el estudio. En este artículo se describirán estos tres modelos: la $R L$ se usa cuando la variable de desenlace o resultado es una variable cuantitativa y se relaciona linealmente con una o más variables independientes cuantitativas, ordinales o dicotómicas. Por ejemplo: si se pretende estimar el volumen de sangrado intraabdominal a partir de diferentes variables como la cinética del trauma, el perímetro abdominal, la edad, el sexo, etc. La Rlog se usa cuando la variable de desenlace o resultado es dicotómica (vivo/muerto, presenta/ausente), a partir de una o más variables independientes, ya sea cuantitativas, ordinales o dicotómicas. MRPC se utiliza cuando se busca determinar el tiempo de supervivencia de un paciente con traumatismo cráneo encefálico donde se considera la edad, el estado de alerta. En investigación clínica y en la atención del paciente en urgencias, los modelos multivariantes ayudan a predecir de modo más confiable el efecto de una maniobra o tratamiento, tomando en cuenta no solo el efecto de un tratamiento, sino de las diferentes variables independientes.
\end{abstract}

Palabras clave: Modelos lineales. Análisis de regresión. Urgencias.

\section{Relevance of multivariate models in emergency medicine}

\section{Abstract}

Multivariate models that are most used in clinical investigation are: linear regression ( $R L)$, logistic regression (Rlog) and Cox proportional risks (MRPC). These models must be mentioned previously in the protocol since the outcome or the expected result of the study. We describe the three models: $L R$ is used when the result variable is quantitative with normal distribution and its related with one or more independent quantitative, ordinal or dichotomous variables. For example, to estimate the intraabdominal blood loss from the current variables: the trauma, age and sex are considered. Rlog is used for dichotomous variables, for example live or dead, present or absent, and are related with one or more variables. These variables could be

\section{Correspondencia:}

${ }^{*}$ Rodolfo Rivas-Ruiz

E-mail: rivasrodolfo@gmail.com
Fecha de recepción: 07-05-2021

Fecha de aceptación: 10-05-2021

DOI: 10.24875/REIE.21000048
Disponible en internet: 30-07-2021

Rev Educ Investig Emer. 2021;3(2):105-112 www.medicinadeemergencias.com

2604-6520 @ 2021 Sociedad Mexicana de Medicina de Emergencias, AC. Publicado por Permanyer México SA de CV. Este es un artículo open access bajo la licencia CC BY-NC-ND (http://creativecommons.org/licenses/by-nc-nd/4.0/). 
quantitative independent variables, ordinals or dichotomous. For example, to predict emergency room mortality from clinical signs. MRPC is used if the result variable is time-person. Is selected when the investigator wants to determinate the survivor time in patients with CET if age and consciousness are considered. For clinical trials at emergency rooms, multivariate models are useful to predict or estimate the most reliable result of a study when other variables are considered.

Key words: Lineal models. Regression analysis. Emergency Medicine.

\section{Antecedentes}

La teoría de la causalidad en medicina es compleja. El desarrollo de una enfermedad o las consecuencias de esta no se deben a una única causa o tratamiento, son el resultado de múltiples variables que pueden condicionar el resultado final. Lo anterior está representado por la máxima de que «no existen enfermedades, existen enfermos", lo cual nos lleva a razonar que existe más de un factor y no solo es un tratamiento el que impacta en el resultado o pronóstico de una enfermedad. Metodológicamente, se pueden hacer distinciones entre grupos mediante la estratificación y/o demarcación de la población de acuerdo con las diferencias en el estado basal y maniobras periféricas que acompañan al tratamiento de interés. Sin embargo, es recomendable ajustar los resultados usando los modelos estadísticos para variables modificadoras del efecto o de confusión como la edad, el peso o algunas comorbilidades, para lo cual estadísticamente se emplean los modelos multivariantes.

Se emplean estos modelos multivariantes cuando queremos saber la relación que existe entre una variable dependiente $(\mathrm{Y})$ y múltiples variables independientes $(\mathrm{X})$.

En la figura 1 se observa la representación esquemática que existe entre las variables independientes (X) que se presentan como factores de riesgo para un evento $(\mathrm{X} 1 \ldots \mathrm{X} 4)$ y otras que se pueden presentar al mismo tiempo (X5... X8), las cuales son covariados 0 confusores. Todas estas variables pueden predecir 0 explicar un desenlace (Y) (Fig. 1).

Un error común es asumir en un estudio de causalidad que la causa es dada por una sola variable. Es decir, en el mundo de la medicina y en el de urgencias se deben de individualizar los casos y tomar en cuenta los antecedentes, el estado actual y severidad de los pacientes, la cinética de la lesión y otros factores asociados.

Por lo tanto, en la investigación clínica también se debe hacer este ejercicio de evaluar la multicausalidad. Por ejemplo, si deseamos evaluar el efecto de la rehidratación con suero oral vs. las soluciones intravenosas para el tratamiento de la deshidratación por gastroenteritis, debemos considerar no solo la maniobra, sino las variables de la demarcación diagnóstica

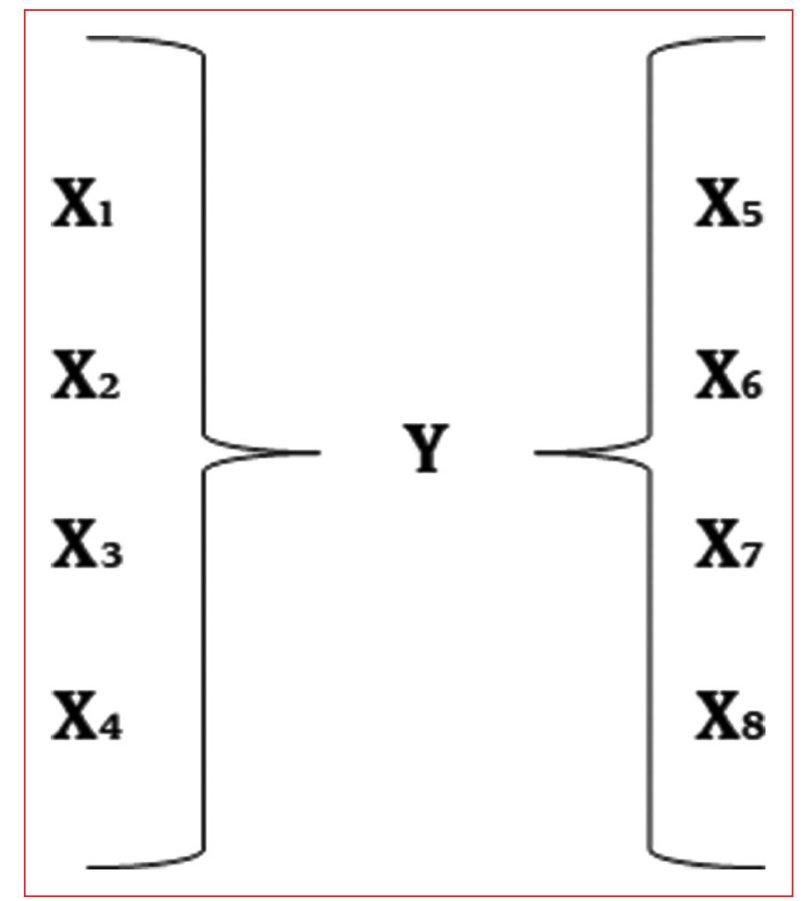

Figura 1. Representación esquemática de la relación de variables en los modelos multivariantes.

y pronóstica presentes en el estado basal ${ }^{1}$. En esta comparación es muy importante considerar la edad del paciente, debido a que los niños más pequeños tendrán una evolución diferente al rehidratarse por su desarrollo intestinal, además de que la rehidratación podría también verse afectada por la composición corporal, el estado nutricional y las comorbilidades. Por lo anterior, para realizar estas ponderaciones estadísticas no bastan los modelos bivariantes, se requieren modelos multivariantes.

\section{Juicio clínico y modelos multivariantes}

El juicio clínico que se realiza en los servicios de urgencias es el mejor ejemplo de un análisis multivariante. Los médicos indican una maniobra u otra a partir del estado basal de los pacientes, de su pronóstico y de la evidencia recabada en los estudios publicados sobre la maniobra que realizar, así como el 
Tabla 1. Supuestos de los modelos multivariantes para su uso en la clínica

\begin{tabular}{|l|l|l|l|l|}
\hline Modelo & Variable dependiente & $\begin{array}{l}\text { Variables } \\
\text { independientes }\end{array}$ & Pregunta clínica & $\begin{array}{l}\text { Resultados } \\
\text { estadísticos }\end{array}$ \\
\hline Regresión lineal & $\begin{array}{l}\text { Cuantitativos con } \\
\text { distribución normal }\end{array}$ & $\begin{array}{l}\text { Cuantitativos con } \\
\text { distribución normal u } \\
\text { ordinales o nominales }\end{array}$ & $\begin{array}{l}\text { ¿Cuáles son los factores que } \\
\text { predicen el sangrado en } \\
\text { volumen (ml)? }\end{array}$ & Beta (ß) \\
\hline Regresión logística & Cualitativa dicotómica & $\begin{array}{l}\text { Cuantitativos o ordinales } \\
\text { o nominales }\end{array}$ & $\begin{array}{l}\text { ¿Cuáles son los factores } \\
\text { predictores de lesión renal } \\
\text { aguda en pacientes con } \\
\text { COVID-19? }\end{array}$ & $\begin{array}{l}\text { Razón de momios 0 } \\
\text { Exp(B) }\end{array}$ \\
\hline $\begin{array}{l}\text { Modelos } \\
\text { proporcionales de Cox }\end{array}$ & Tiempo-persona & $\begin{array}{l}\text { Cuantitativos o ordinales } \\
\text { onominales }\end{array}$ & $\begin{array}{l}\text { ¿Cuáles son los factores } \\
\text { predictores la supervivencia } \\
\text { de los pacientes con IAM? }\end{array}$ & $\begin{array}{l}\text { Híbrido de riesgo (HR) } \\
\text { log ß }\end{array}$ \\
\hline
\end{tabular}

COVID-19: enfermedad por coronavirus 2019; IAM: infarto agudo de miocardio.

tamaño del efecto esperado tras la administración de la maniobra ${ }^{2}$.

Un ejemplo donde se hace patente el juicio clínico es la intubación de pacientes con enfermedad por coronavirus 2019 (COVID-19). Resulta conocido que los pacientes infectados con coronavirus 2 del síndrome respiratorio agudo grave (SARS-CoV-2) son susceptibles a presentar insuficiencia respiratoria. Sin embargo, no hay suficiente evidencia respecto a la eficacia para recomendar la intubación orotraqueal o cánulas de alto flujo o algún otro método no invasivo ${ }^{3}$.

Los médicos deciden el tipo de ventilación a partir de múltiples variables como: los datos de dificultad respiratoria, antecedentes, oximetría de pulso, exploración física, edad o comorbilidades, entre otros. Este juicio, que se va mejorando con la experiencia, se realiza de forma inmediata y no solo como "acto de magia" o que se debe al "ojo clínico". Sin embargo, en realidad se basa en modelos multivariantes dentro de la mente del médico y en la mayoría de los casos en la lectura de estudios clínicos de casos similares.

Por lo anterior es que consideramos importante enunciar los tipos más comunes de modelos multivariantes y de los supuestos que se requieren para llevarse a cabo, con el fin de reforzar el juicio clínico de los colegas de los servicios de urgencias durante la toma de decisiones.

\section{Supuestos de los modelos multivariantes}

Los supuestos son las reglas matemáticas que deben cumplir los modelos para ser adecuados y dar resultados que sean reproducibles universalmente. Es por esta razón que un buen clínico debe conocer cuáles son estos supuestos que los hacen adecuados (pertinentes) para contestar cada una de las preguntas clínicas.

Cada uno de los modelos tiene sus supuestos particulares. Sin embargo, podríamos mencionar algunos importantes, como el que las variables cuantitativas que vayan a ser usadas en los modelos deben de tener distribución normal. En modelos de regresión logística o de Cox se debe contar con un número suficiente de pacientes en cada uno de los grupos estudiados. Se recomienda que se tengan de 10 a 20 eventos por cada una de las variables que se van a incluir en el modelo.

Si bien es cierto que los modelos multivariantes tienen similitudes entre ellos, es importante conocer que la precisión del resultado del estudio depende de la selección de la variable dependiente. Es decir, qué es lo que se requiere impactar.

Otro punto importante en los modelos multivariantes es el principio de parsimonia, el cual indica que se deben de usar el menor número de variables para explicar un fenómeno, siendo la mayoría de ellas útiles para realizar la predicción final en el escenario clínico ${ }^{4}$.

Los supuestos de los modelos multivariantes más comúnmente usados en la clínica se presentan en la tabla 1.

Las variables independientes también se pueden denominar variables predictoras o explicativas, ya que estas son las que podemos manipular o las que pueden variar dependiendo de las características de los pacientes, a estas maniobras se les atribuye que suceda un fenómeno. Las variables dependientes son las variables de resultado o de desenlace, debido a que estas variables son la consecuencia de todas las variables independientes. 


\section{Regresión lineal}

\section{Antecedentes}

La regresión lineal tiene por lo menos 200 años en uso. Diferentes eruditos como Pearson y Galton, por medio de experimentos y observaciones, han contribuido con las características y supuestos de este modelo que en la actualidad conocemos. El análisis de regresión lineal tiene su origen en los análisis de correlación de Pearson ${ }^{5}$.

La regresión lineal se emplea cuando queremos saber la relación que existe entre una variable desenlace (dependiente) continua como sangrado, hemoglobina o glucosa, con variables predictoras (independientes) como la edad o el sexo, entre otras características propias de los que deseemos estudiar.

Conociendo la relación que tienen estas variables podremos predecir qué pasará en el desenlace cuando se modifican las diferentes variables predictoras $\sin$ necesidad de confirmarlo experimentalmente. Sin embargo, para que este cálculo sea válido, debemos respetar los supuestos que mencionaremos a continuación.

\section{Supuestos}

La regresión lineal se emplea cuando se quiere conocer la relación entre una variable independiente de tipo cuantitativa, ordinal o dicotómica y una variable dependiente cuantitativa.

- La variable dependiente debe de ser cuantitativa y tener distribución normal o un comportamiento gaussiano. Esto es debido a que este modelo utiliza las distribuciones $F$ (de Snedecor) o t (de Student) para evaluar la significancia estadística.

- Debe de existir una relación lineal entre ambas variables, ya sea positiva o negativa (mientras una variable aumenta la otra también o mientras una disminuye la otra incrementa). A este fenómeno se le denomina proporcionalidad.

\section{Expresión matemática}

Sabiendo lo anterior, la forma de aplicación de la regresión lineal no es más que una fórmula sencilla de la ecuación de la recta:

$$
y=\beta_{0}+\beta_{1} x
$$

En donde y es igual a la variable dependiente, $\beta_{0}$ corresponde a la ordenada de origen o intersección, es decir el valor de y cuando $x$ vale cero, y $\beta_{1} x$ es la pendiente o razón con la que el fenómeno del efecto está cambiando.

Lo importante de este aspecto es conocer que la regresión lineal puede ser simple cuando se busca la relación entre dos variables (1 variable dependiente vs. 1 variable independiente) y múltiple cuando se incluyen más de dos variables (u1na variable dependiente vs. $\geq 2$ variables independientes) y, por lo tanto, la expresión matemática cambia solo adicionado los parámetros de las variables independientes.

La ordenada al origen es la que nos indica el número de variables independientes que existen en nuestra regresión lineal, por lo que cuando se trate de una regresión lineal múltiple vamos a tener más de una $\mathrm{m}$ $y$ una $b$.

De tal forma que la regresión lineal simple se expresa:

$$
y=\beta_{0}+\beta_{1} x+\varepsilon
$$

Donde $\beta$ representa las estimaciones de parámetros lineales que se deben calcular y $\varepsilon$ representa los términos de error.

Y la múltiple podría expresarse:

$$
y=\beta_{0}+\beta_{1} x_{1}+\beta_{2} x_{2}+\beta_{3} x_{3}+.+\beta_{p} x_{p}+\varepsilon
$$

Las $\beta i$ ( $i>0$ ) son los parámetros respectivos de cada variable independiente y $p$ es el número de parámetros independientes que considerar en la regresión ${ }^{6}$.

\section{Juicio clínico}

En la clínica podemos emplearlo cuando queramos predecir o estimar, por ejemplo, el número de horas de estancia en urgencias, cantidad de hemoderivados requeridos, torundas o todo aquello que se pueda contar de forma cuantitativa, a partir de una variable que nosotros podamos controlar ${ }^{7,8}$.

En el área de urgencias médicas (AUM) se puede emplear para determinar, por ejemplo, la relación entre el tiempo transcurrido hasta la administración de analgésicos y la duración de la estancia en el servicio de urgencias $^{9}$; en dicho ejemplo la variable predictora es el tiempo que transcurre hasta la administración de analgésicos y la variable desenlace es la duración de la estancia en el AUM.

\section{Regresión logística}

La mayoría de los estudios clínicos suelen buscar una resolución dicotómica (presente/ausente, vivo/muerto, enfermo/sano). Esto se debe quizá a que el juicio clínico posterior a una evaluación clínica siempre termina en 
una acción o no acción. El análisis multivariante cuando se quiere estudiar una variable dependiente dicotómica se hace con el modelo de regresión logística múltiple. Este es un análisis multivariante explicativo y predictivo ampliamente usado en la investigación clínica, epidemiológica y algunas veces en la básica.

\section{Antecedentes}

La regresión logística es uno de los instrumentos estadísticos más expresivos y versátiles de los que se dispone para el análisis de datos en clínica y epidemiología. Es el resultado del trabajo de diversos matemáticos desde el siglo xiv, pero su forma moderna fue principalmente desarrollada por Joseph Berkson en 1940 y ampliamente aplicada a los análisis de datos epidemiológicos por David Cox entre 1950 y 1960.

Su uso en investigación clínica se universaliza y expande desde principios de los 80 debido, especialmente, a las facilidades informáticas con las que se cuenta desde entonces ${ }^{10}$.

\section{Supuestos}

- La variable dependiente o de resultado es cualitativa dicotómica, por lo cual sus valores son mutuamente excluyentes.

- Las variables independientes o predictoras pueden ser cuantitativas o cualitativas (ordinales o dicotómicas).

- Se deben utilizar variables predictoras únicamente cuando hayan demostrado relación en el análisis bivariante con respecto a la variable dependiente 0 que la asociación sea biológicamente plausible.

\section{Expresión matemática}

$\ln [y /(1-y)]=\beta_{0}+\beta_{1} x_{1}+\beta_{2} x_{2}+\beta_{3} x_{3}+\beta_{4} x_{4}+\beta_{5} x_{5}$ Donde y es el logaritmo natural (In) de las probabilidades (odds), $\beta_{0}$ es la constante de regresión o intersección, es decir el valor de y cuando $x$ es igual a cero, lo que significa la probabilidad de la enfermedad o el resultado de interés cuando no se considera ningún factor de riesgo (y se corresponde con la prevalencia de una enfermedad). $\beta$ es el coeficiente de regresión de cada variable y se refiere a la magnitud de cambio en y por cada unidad de cambio en $\mathbf{X}^{11}$.

Como podemos observar, esta ecuación se corresponde con la ecuación de la recta vista en el modelo de regresión lineal. Sin embargo, en el modelo de regresión logística se utiliza la función logarítmica de la ecuación. Con el uso de los logaritmos se puede
Tabla 2. Modelo de regresión logística múltiple para predecir lesión renal aguda en pacientes con infarto agudo de miocardio

\begin{tabular}{|l|c|c|}
\hline Variables & OR $\operatorname{Exp}(ß)$ & IC $95 \%$ \\
\hline Edad (por cada 10 años de edad) & 1.04 & $1.02-1.05$ \\
\hline Hipertensión & 2.51 & $1.62-3.87$ \\
\hline Enfermedad renal crónica & 3.52 & $2.01-6.12$ \\
\hline Uso de furosemida & 1.02 & $1.02-1.03$ \\
\hline
\end{tabular}

OR: odds ratio, razón de momios; IC 95\%: intervalo de confianza $95 \%$. Adaptada de Wang, et al..$^{12}$

evaluar la variable en forma dicotómica (incremento de riesgo o disminución de riesgo), de la regresión logística se obtiene el exponente de $\beta$ de cada una de las variables independientes que es lo mismo que la $O R$ (odds ratio) o la RM (razón de momios), lo cual a su vez es equivalente a la RM que se obtiene en una tabla de $2 \times 2\left(\chi^{2}\right)$ en un análisis bivariante.

La interpretación de una RM se realiza multiplicando la RM por la prevalencia de la enfermedad como ejemplificamos más adelante.

\section{Juicio clínico}

Este es uno de los modelos favoritos por los clínicos, el cual se puede adaptar a una gran variedad de preguntas donde el resultado sea dicotómico, por ejemplo, estudios en neonatos donde se requiere el ajuste por edad gestacional, sexo, etc. ${ }^{13}$

Otro ejemplo es un estudio retrospectivo en el que se incluyeron a los pacientes con diagnóstico de infarto agudo de miocardio (IAM) ingresados en un servicio de urgencias, los cuales fueron evaluados hasta su egreso, buscando como variable dependiente dicotómica la lesión renal aguda (LRA) ${ }^{12}$. Las variables que demostraron significancia estadística en el análisis bivariante fueron: edad, enfermedad renal crónica (ERC), hipertensión arterial (HTA) y uso de furosemida. Por lo tanto, estas variables fueron incluidas en un modelo de regresión logística múltiple (RLOG) para evaluar la interacción entre estas variables, cuánto contribuyen de manera independiente o cuánto se asocian con el desarrollo de LRA. En el modelo de análisis multivariante de regresión logística para mostrar la relación independiente de cada variable independiente con la variable resultado, se calcularon las OR como medidas de asociación y los intervalos de confianza correspondientes, como podemos ver en la tabla 2. 
Se puede concluir que por cada 10 años que aumenta la edad de los pacientes, la presencia de uso de furosemida y la presencia de comorbilidades como hipertensión o enfermedad renal crónica aumentan el riesgo de presentar LRA en pacientes con IAM.

Debido a que la prevalencia de LRA en este estudio es del $10 \%$, el que el paciente presente hipertensión eleva el riesgo 2.51 veces.

El riesgo final de LRA es el resultado de la suma de las variables que fueron significativas en el modelo. LRA = edad (1.04) + HTA (2.51) + ERC (3.52) + furosemida (1.02).

Es importante recordar que estas son asociaciones estadísticas y no necesariamente implican causalidad.

\section{Riesgos proporcionales de Cox}

El modelo de Cox se emplea con mucha frecuencia en estudios clínicos en los últimos años, debido a que es un análisis que ayuda a predecir el riesgo de que ocurra un evento con la finalidad de evaluar la supervivencia de dicho evento en un determinado tiempo. Es decir, se utiliza para evaluar cuántas personas sobreviven o presentan un evento en determinado tiempo. A pesar de que se llama análisis de supervivencia, puede usarse cuando el desenlace es dicotómico, aun cuando no sea vive o fallece, sino, por ejemplo, cuando se desea evaluar la aparición de complicaciones en determinado momento en el tiempo. Este modelo es muy usado cuando lo que se desea conocer es la diferencia de tiempo entre una maniobra y otra. Este análisis utiliza una unidad de tiempo-persona, la cual puede ser expresada como riesgo.

Estos modelos han sido muy usados en especialidades como la oncología para conocer la supervivencia a un determinado tiempo y la comparación de una maniobra frente a otra ${ }^{14,15}$.

\section{Antecedentes}

El modelo de riesgos proporcionales de Cox (MRPC), también denominado análisis de supervivencia, es un tipo de análisis multivariante que fue creado en 1972 por el estadístico inglés Sir David R. Cox, con la finalidad de modelar los riesgos que podrían afectar la supervivencia en una población.

EI MRPC expresa la función de riesgo considerando múltiples variables explicativas para predecir un desenlace dicotómico (vivo/muerto) que se mide a lo largo del tiempo, es decir, pronostica la probabilidad de que ocurra un evento de interés en determinado tiempo. Sin embargo, también se debe tomar en cuenta los datos "censurados", que son aquellos datos faltantes que desaparecen en el transcurso del estudio o las observaciones que no desencadenan el evento de interés antes de finalizar el estudio ${ }^{16}$.

\section{Supuestos}

- La proporción de los riesgos de un caso a otro no debe variar en función del tiempo.

- Se requiere un desenlace dicotómico (progresión o no de una enfermedad a lo largo del tiempo).

- Los datos censurados o faltantes no se relacionan con la probabilidad de que ocurra el evento desenlace de interés.

La interpretación del MRPC se realiza por medio de la medida de riesgo hazard ratio $(\mathrm{HR})$, sus intervalos de confianza y valor de significancia p. Sin embargo, también se utilizan representaciones gráficas como las curvas de supervivencia y diagramas de bosque (forest plot).

Las curvas de supervivencia se utilizan con la finalidad de observar cuántas personas sobreviven o presentan un desenlace de interés en tiempo determinado.

Los forest plots exponen los HR y sus intervalos de confianza de cada variable predictora con la finalidad de observar si existe una asociación de dichas variables con el desenlace de interés ${ }^{16}$.

\section{Expresión matemática}

La función del riesgo se expresa mediante la siguiente ecuación:

$\lambda(t ; Z i(t))=\lambda 0(t) e \beta{ }^{\prime} Z i(t) \circ \lambda i(t)=\lambda 0(t)$ e xi $(t) \beta$

Donde $\lambda_{0}(t)$ es una función de riesgo no negativa $y$ sin especificar, común a todos los sujetos del estudio, es decir «función de riesgo base»; $\beta$ es el vector de coeficientes del modelo y Z, covariantes que tienen un efecto multiplicativo en la función del riesgo.

La medida de riesgo que se emplea para conferir un desenlace se denomina híbrido de riesgo o hazard ratio (HR). El HR se refiere a la probabilidad que tiene un grupo de individuos de presentar un evento de interés en comparación con otros grupos, por ejemplo, un grupo de referencia en un tiempo determinado. Dicha medida de riesgo se expresa de la siguiente manera:

HR (sucesos ocurridos en el instante t en el grupo 1/ sujeto en riesgo en el instante t en el grupo 1)/(sucesos ocurridos en el instante t en el grupo 2)/(sujeto en riesgo en el instante $t$ en el grupo 2) $=h 1 / h 2$. 
Además de los HR, se deben obtener los intervalos de confianza y el valor de $\mathrm{p}$ de la $\mathrm{X}^{2} \mathrm{de}$ Wald para la interpretación del riesgo y probar la significación del efecto medido. El valor de $p$ de Wald se interpreta de la misma manera que el valor de $p$ de cualquier otra prueba estadística.

\section{Juicio clínico}

Supongamos que ingresa un paciente al AUM por dolor torácico (DT). Es de conocimiento que el DT es el principal síntoma de los pacientes con sospecha de síndrome coronario agudo (SCA) y el primordial motivo para que en servicio de urgencias les determinen la elevación de troponina ( $T n c)$, ya que estos indicadores permiten descartar un SCA en pacientes con DT, facilitan el diagnóstico de infarto de miocardio (IAM) y contribuyen en llevar a cabo una mejor estrategia terapéutica. Por otra parte, cabe mencionar que existen otros pacientes que ingresan al AUM y no expresan DT, pero sí presentan elevación de Tnc y de igual manera los puede condicionar a desencadenar IAM 0 SCA. Asimismo, no se sabe cuál es el pronóstico en un seguimiento a un año de los pacientes que presentan DT y Tnc elevada vs. los que no lo presentan.

Para resolver el cuestionamiento anterior se revisó un estudio de cohorte retrospectivo, en el cual se incluyeron los pacientes que ingresaban al AUM por cualquier causa, se les determinó las Tnc al ingreso y seis horas después. Posteriormente, se agrupó a la población en cuatro grupos diagnósticos: aquellos que presentan DT con Tnc elevada, ausencia de DT con Tnc elevada, DT sin Tnc elevada y ausencia de DT $\sin$ Tnc elevada ${ }^{17}$.

Para evaluar el pronóstico, se buscó como variable dependiente la mortalidad por cualquier causa a los 30 días después del ingreso al AUM y al año de seguimiento.

En el análisis bivariante, las variables que mostraron significancia estadística fueron la edad, sexo, factores de riesgo cardiovasculares, comorbilidades (antecedentes de enfermedades cerebrovasculares [ECV]), anemia, hiperglucemia, saturación de oxígeno, creatinina, presión arterial sistólica y hemoglobina.

Por tanto, los cuatros grupos diagnósticos fueron ajustados por las siguientes variables mediante el modelo de Cox.

En la tabla 3 se puede observar como la edad, sexo y los antecedentes de insuficiencia cardiacay de ECV, asícomo lapresencia de anemia, hiperglucemiay creatinina elevada, fueron factores independientes relacionados con mayor mortalidad. Sin embargo, se puede concluir que los
Tabla 3. Análisis multivariante por regresión de Cox para mortalidad a un año

\begin{tabular}{|c|c|c|c|c|}
\hline & \multirow[t]{2}{*}{ HR } & \multicolumn{2}{|c|}{ IC $95 \%$} & \multirow[t]{2}{*}{ p } \\
\hline & & Inferior & Superior & \\
\hline Edad (años) & 1.039 & 1.028 & 1.050 & $<0.001$ \\
\hline Hombres & 1.305 & 1.011 & 1.686 & 0.041 \\
\hline $\begin{array}{l}\text { Antecedentes de } \\
\text { ECV }\end{array}$ & 1.498 & 1.099 & 2.043 & 0.011 \\
\hline PA Sistólica & 0.989 & 0.985 & 0.993 & $<0.001$ \\
\hline Sat. $\mathrm{O}_{2}$ & 0.961 & 0.943 & 0.979 & $<0.001$ \\
\hline Primera glucemia & 1.002 & 1.001 & 1.004 & 0.001 \\
\hline Primera creatinina & 1.173 & 1.083 & 1.272 & $<0.001$ \\
\hline Hemoglobina & 0.909 & 0.862 & 0.958 & $<0.001$ \\
\hline $\begin{array}{l}\text { Grupos } \\
\text { diagnósticos } \\
\text { DT y Tnc no } \\
\text { elevada } \\
\text { No DT y Tnc no } \\
\text { elevada }\end{array}$ & $\begin{array}{c}1 \\
\text { (Ref.) } \\
1.943\end{array}$ & 1.225 & 3.081 & 0.005 \\
\hline $\begin{array}{l}\text { DT y Tnc } \\
\text { elevada }\end{array}$ & 3.856 & 2.441 & 6.093 & $<0.001$ \\
\hline $\begin{array}{l}\text { No DT y Tnc } \\
\text { elevada }\end{array}$ & 5.130 & 3.291 & 7.996 & $<0.001$ \\
\hline
\end{tabular}

IC 95\%: intervalo de confianza del 95\%; ECV: enfermedad cerebrovascular; PA: presión arterial, Sat. $\mathrm{O}_{2}$ : saturación arterial de

oxígeno; Ref: referencia; HR: hazard ratio; DT: dolor torácico; Tnc: troponina.

pacientes con Tnc elevada sin DT tuvieron peor supervivencia (HR: 5,130; Intervalo de confianza del 95\%: $3,291-7,996 ; p<0.001)$ con respecto a los pacientes con DT con Tnc no elevada ${ }^{17}$.

Además, los niveles altos de Tnc en estos pacientes sirven para identificar un alto riesgo para desencadenar SCA y contribuyen en la mejora de métodos diagnósticos para intentar mejorar el pronóstico y, aunado a esto, su calidad de vida.

\section{Conclusiones}

Los modelos multivariantes son aplicados por los médicos por medio del juicio clínico al hacer predicciones sobre el pronóstico o curso clínico de la enfermedad posterior a la administración de los diferentes tratamientos, el cual se hará con base en el análisis de múltiples características del paciente, las cuales están presentes en el estado basal (comorbilidades, etapa de la enfermedad o estado nutricional, indicaciones o contraindicaciones de la maniobra, etc.), la 
administración conjunta del tratamiento con otras medidas generales o sintomáticas (tipo de solución, uso de aminas, tipo de dieta, uso de antibiótico, transfusión, etc.).

En la investigación clínica los modelos multivariantes son usados para evaluar los efectos de una maniobra, ajustada por el efecto simultáneo de otras variables que pueden modificar el efecto final. Dependiendo del tipo de variable que se va a predecir se utiliza ya sea una regresión lineal, logística o de riesgos proporcionales de Cox.

Los modelos multivariantes son usados en la mayoría de los estudios de investigación que asumen causalidad en la clínica' ${ }^{18}$; por lo anterior es indispensable su estudio para mejorar el juicio clínico en el día a día por su aplicación directa en el quehacer diario en los servicios de urgencias médicas.

\section{Financiamiento}

El presente trabajo no ha recibido ninguna beca específica de agencias de los sectores públicos, comercial o sin ánimo de lucro.

\section{Conflicto de intereses}

Los autores declaran no tener conflicto de intereses.

\section{Responsabilidades éticas}

Protección de personas y animales. Los autores declaran que para esta investigación no se han realizado experimentos en seres humanos ni en animales.

Confidencialidad de los datos. Los autores declaran que en este artículo no aparecen datos de pacientes.

Derecho a la privacidad y consentimiento informado. Los autores declaran que en este artículo no aparecen datos de pacientes.

\section{Bibliografía}

1. Hartling L, Bellemare S, Wiebe N, Russell K, Klassen TP, Craig W. Oral versus intravenous rehydration for treating dehydration due to gastroenteritis in children. Cochrane Database Syst Rev. 2006;19;2006(3): CD004390.

2. Talavera JO, Roy-García IA, Pérez-Rodríguez M, Palacios-Cruz L, Rivas-Ruíz R. Back to the clinic. Methods II. The Architecture of Clinical Research. Subject interaction, maneuvers and disease throughout time. Gac Med Mex. 2020;156(5):430-8.

3. Montrief T, Ramzy M, Long B, Gottlieb M, Hercz D. COVID-19 respiratory support in the emergency department setting. Am J Emerg Med. 2020;38(10):2160-8.

4. Roy-García I, Rivas-Ruiz R, Pérez-Rodríguez M, Palacios-Cruz L. Correlación: no toda correlación implica causalidad. Rev Alerg Mex. 2019;66(3):354-60

5. Wells CK, Feinstein AR, Walter SD. A comparison of multivariable mathematical methods for predicting survival-III. Accuracy of predictions in generating and challenge sets. J Clin Epidemiol. 1990;43(4):361-72.

6. Palacios-Cruz L, Pérez M, Rivas-Ruiz R, Talavera JO. Investigación clínica XVIII. Del juicio clínico al modelo de regresión lineal. Rev Med Inst Mex Seguro Soc. 2013;51(6):656-61.

7. Vázquez E, Ledeneva Y, García-Hernádez RA. Learning relevant models using symbolic regression for automatic text summarization. Comp y Sist. 2019;23 1):127-41.

8. Gujarati DN. Linear regression model: Distribution theory and hypothesis testing. En: Gujarati DN. Linear regression: A mathematical introduction. SAGE Publications; 2019. pp. 59-90.

9. Hughes JA, Brown NJ, Chiu J, Allwood B, Chu K. The relationship between time to analgesic administration and emergency department length of stay: A retrospective review. J Adv Nurs. 2020;76(1):183-90.

10. Hess AS, Hess JR. Logistic regression. Transfusion. 2019;59(7):2197-8.

11. Berea-Baltierra R, Rivas-Ruiz R, Pérez-Rodríguez M, Palacios-Cruz L, Moreno J, Talavera JO. Investigación clínica XX. Del juicio clínico a la regresión logística múltiple. Rev Med Inst Mex Seguro Soc. 2014;52(2): 192-7.

12. Wang C, Pei YY, Ma YH, Ma XL, Liu ZW, Zhu JH, et al. Risk factors for acute kidney injury in patients with acute myocardial infarction. Chin Med $\mathrm{J}$ (Engl). 2019;132(14):1660-5.

13. Chiclla MA, Castillo K, Arias JP. Predictores perinatales de APGAR persistentemente bajo a los 5 minutos en un hospital peruano. Rev Fac Med Hum. 2021;21(1):40-9.

14. Ramírez-Torres N, Pérez-Puentes A, Moctezuma-Meza C, Victoria-Ayala R, Ortiz-Rodríguez K, Valenzuela-Martínez LA, et al. Neoadjuvant epirubicin with or without sequential docetaxel in treatment of advanced breast cancer: assessment of pathological complete response and chemotherapy tolerability. Cir Cir. 2019;87(1):59-68.

15. Zitle-García EJ, Sauceda-Valenzuela AL, Ascencio-Montiel IJ, García-Paredes J. Tumores malignos condicionantes de invalidez en el Instituto Mexicano del Seguro Social. Rev Med Inst Mex Seguro Soc. 2018;56(2):173-9.

16. Pérez-Rodríguez $M$, Palacios-Cruz L, Moreno J, Rivas-Ruiz R, Talavera JO. Investigación clínica XXII. Del juicio clínico al modelo de riesgos proporcionales de Cox. Rev Med Inst Mex Seguro Soc. 2014:52(4):430-5.

17. González-del-Hoyo M, Cediel G, Carrasquera A, Bonet G, Consuegra-Sánchez L, Bardají A. Implicaciones diagnósticas y en el pronóstico de la elevación de troponina en ausencia de dolor torácico en pacientes atendidos en urgencias. Emergencias. 2018;30:77-83.

18. Sagaró del Campo N, Larisa Zamora M. Técnicas estadísticas multivariadas para el estudio de la causalidad en medicina. Rev Ciencias Médicas. 2020;24(2):e4029. 